\title{
Utilization of Metal Additive Manufacturing (AM) in Precision Oriented Mechanical Part Production
}

\author{
Hamidreza Javidrad \\ Department of Mechanical Engineering \\ Iran University of Science and Technology \\ Tehran, Iran
}

\author{
Mostafa Larky \\ Department of Mechanical Engineering \\ Iran University of Science and Technology \\ Tehran, Iran
}

\begin{abstract}
Metal additive manufacturing (AM) is an innovative technology that opens up new visions in any industrial field. This technology is growing rapidly, entering various industries every day. After over 30 years from the introduction of metal additive manufacturing into the industrial world, the today's demand is to make mechanical parts with high precision by metal additive manufacturing. Until now, methods like high precision grinding or micro-machining processes are used to make more precise dimensions in parts. Those methods have their own limits and deficiencies that could be eliminated with additive manufacturing. In this paper, attempts are made to compare conventional precise processes with metal additive manufacturing from different aspects. Surface finish in these two categories is discussed and some advice for improving the surface quality of metal additive manufacturing parts is proposed. Applicability and utilization of metal additive manufacturing as a precise metal fabrication method in various industries with examples are also discussed.
\end{abstract}

Keywords: Additive manufacturing, precision manufacturing, inkjet, benchmark, micro fabrication.

\section{INTRODUCTION}

According to ASTM, additive manufacturing (AM) is defined as the process of joining materials to make objects from 3D model data, usually layer upon layer, as opposed to subtractive manufacturing methodologies [1]. With the arrival of revolutionary metal AM, every industry is now looking for new applications of this technology in their field. AM has shown great potential in almost every high-tech industry such as aerospace, electronics, biomechanics, MEMS, etc., and has provided a strong tool to build near net shape parts with any level of complexity.

Fabrication of smaller parts with higher precision has determined as a new demand in serious industries. Precision manufacturing refers to any process that leads to fabricate micro and nano-scale parts and features with high accuracy. Precision manufacturing is one of those interesting fields that could be applied to a wide range of industries such as porous structures in medical implants, micro-scale heat exchangers, parts with embedded structures, reference blocks, etc. Precision manufacturing processes capability depends on several parameters. To fabricate precision parts by means of AM, effective process parameters should be identified. Level of part accuracy depends on process planning and parameters involved. In the following sections, a comparison between $\mathrm{AM}$ and other precision methods are discussed. Then, some solutions for AM process deficiencies are presented. Last, a brief review on micro fabrication by means of $\mathrm{AM}$ is presented.

\section{COMPARISON BETWEEN}

\section{ADDITIVE AND SUBTRACTIVE \\ PRECISION MANUFACTURING}

Subtractive precision manufacturing processes are divided into two categories: (1) conventional methods (i.e. micromachining, micro-scale abrasive processes, etc.) and (2) nonconventional (i.e. micro-electrical discharge machining $(\mu \mathrm{EDM})$ and micro-electrochemical machining $(\mu \mathrm{ECM})$, laser micro-machining, etc.) processes [2]. Although each of these processes has its own limitations; nevertheless, most of them are not capable of producing complex parts. Moreover, processes such as micro milling need precise tools and equipment which are expensive and hard to manufacture.

In contrast to other precision manufacturing processes, AM has the capability of building high level complex parts but its surface quality and tolerance is not satisfying [3]. Figure 1 shows poor resolution of boundaries due to layer by layer deposition. Subsequently, parts fabricated by AM are required post-processing treatments to enhance micro structural and surface properties. By combination of AM and conventional or non-conventional machining processes, high precision and accuracy and fine surface finish could be achieved. Wang et al. [4] combined directed energy deposition (DED) process with CNC machining to attain better accuracy. Such combinations are known as hybrid manufacturing. This could improve mechanical behaviors such as fatigue life [5] and help fabricate more efficient parts. However, it may lead to some difficulties like part distortion observed during fused deposition modeling (FDM) of the second part on a premanufactured machined part due to thermal gradient [6]. Part distortion is severely occur in metallic parts. For example, Afazov et al. [7] reported a turbine blade which is manufactured by powder-bed AM processes had approximately $\pm 300 \mu \mathrm{m}$ distortion. This number could be reduced through a compensation methodology during designing procedure [7]. 
(a)

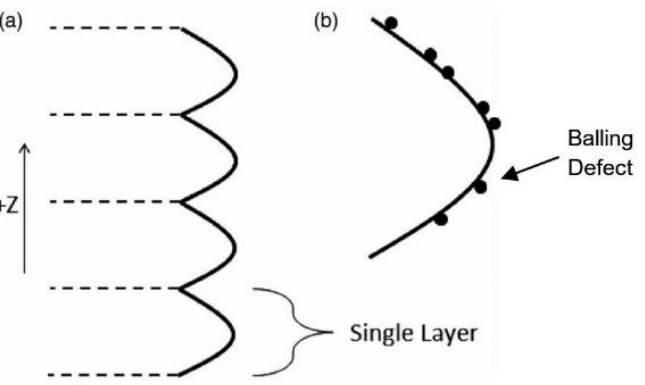

Figure. 1 AM deposited layers representing (a) poor resolution in boundaries and (b) balling defect on a single layer.

Manufacturing processes could be managed in a way that minimizes waste of material and time as much as desirable accuracy and precision [3]. However, AM has high potential to be combined with other manufacturing processes such as electron discharge machining (EDM), electrochemical machining, grinding, etc., to obtain better surface finish and higher accuracy. By this means, AM could be employed just for complex features of a part which are impossible or hard to be made by other processes. An attempt done by Butzhammer et al. [8]. They employed laser beam melting (LBM) to build some functional features on sheet metals. According to their comparison between hybrid manufacturing and purely additive and subtractive manufacturing, it is concluded that hybrid manufactured parts could reach almost $90 \%$ of mechanical properties of subtractive manufactured part, while it could also save raw material and manufacturing time as well as better surface finish.

Precision manufacturing may refer to micro-scale part fabrication. These parts have used in micro-electromechanical systems (MEMS), micro-opto-electro-mechanical systems (MOEMS), microelectronic products, micro-optical electronics systems (MOES) and any applicable combination [9]. New AM methods have this capability to facilitate microscale part production. Many related works are done in this field and could be found in the literature [10-12]. For example, in [11], authors have believed that no single AM process is able to satisfy high resolution, high purity and intricate geometry and current methods still struggle to guarantee high quality products. They reviewed recent trends and latest micro-scale fabrication processes by means of AM. As shown in figure 2, every AM-based process has its precision scale depend on process characteristics, material type, physical state of the material, etc. These boundaries are not rigid and could change as technology grows. Two key methods in this diagram are inkjet and laser-induced forward transfer (LIFT) which are very promising and flourishing.

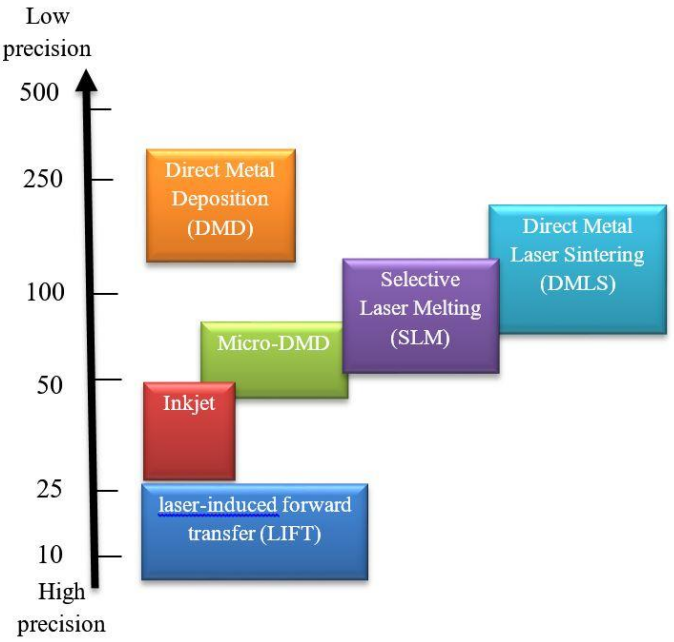

Figure. 2 Least dimensional accuracy achieved by metalbased AM processes until now (highly depend on material and process parameters).

\subsection{INKJET}

One of the new AM methods which has the capability of micro-scale production is droplet printing better known as inkjet. In this method molten metal droplets are dropped from specific location above the substrate and cool down during free fall before solidifying in a few nanosecond as soon as touching the substrate. This strategy provides a situation which eliminate melt pool problems and spreading melts [13]. However, positioning where droplets land, jet height, temperature and other parameters require accurate control to achieve precise shape. Figure 3 demonstrates schematic of two different types of inkjet system. Drop on-demand (DOD) process type has better controllability and accuracy due to singularity and independency of each droplet, however, it is more time consuming in comparison to continuous system. Researchers in this field have mainly focused on droplet geometry and its heat transfer and control to achieve higher precision as well as higher production rate. It should be noted that inkjet processes have almost no material waste, therefore, high value metals like gold could be widely used. MEMS technology have significantly benefited from this production method.

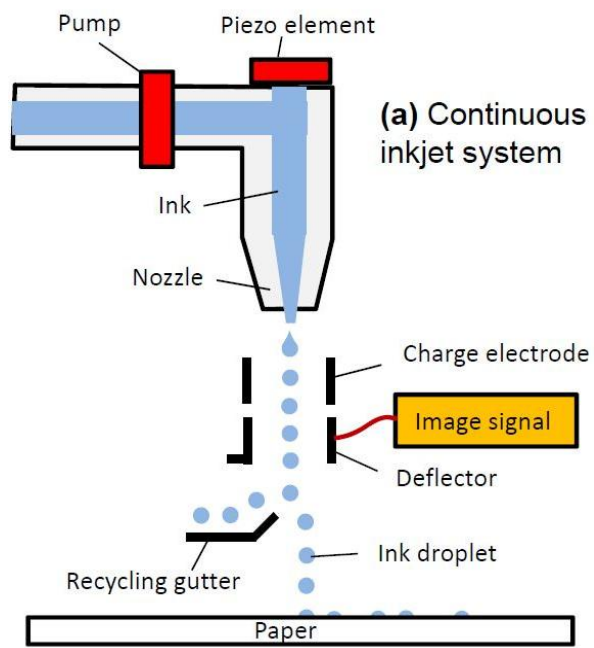


(b) On-demand

inkjet system

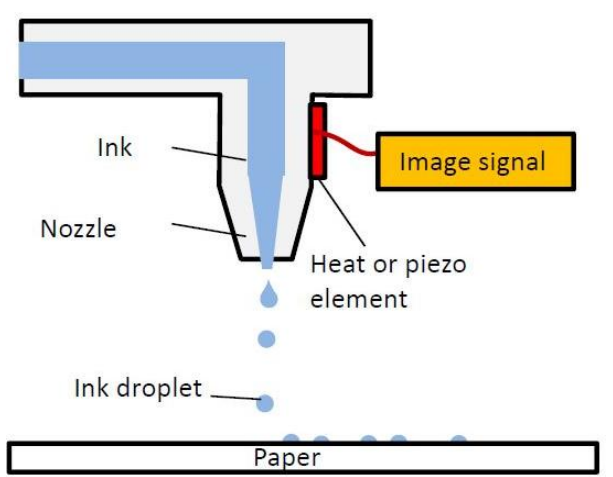

Figure. 3 Schematics demonstrating: (a) a continuous inkjet printer; (b) an on-demand inkjet printer [14].

From a closer look at the droplet head in figure 4, many functions such as heating source, orifice, electrode charger, etc. could be seen. From the top, depends on type of feed, wire or powder are fed to the melting chamber. Melting chamber is built of ceramics or high melting point metals to withstand high temperature. Heating source melt the metal inside and then molten metal pour though control orifice. Charging electrode mainly used in continuous systems and cut the stream into single droplets and make it possible to change landing position of droplets by changing the charge voltage [15].

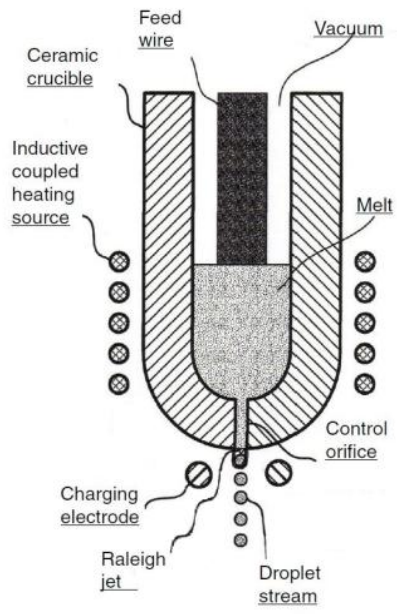

Figure. 4 Metal droplet generator head design using wire feed system [16].

\subsection{LASER-INDUCED FORWARD TRANSFER (LIFT)}

LIFT process is a high precision direct-write additive manufacturing process which has the ability of fabricating 3D micro-parts from thin metal plate. Figure 5 shows the schematic of this process. During the process, a ultra-violet laser pulse in a few picosecond causes ablation through a thin metallic plate which is coated on a carrier substrate (usually glass slide) [10]. This means oxidation and phase changes minimized due to elimination of melting pool and heat affected zone (HAZ) [10]. Accurate positioning of droplets is highly depending on gap distances, therefore, all gap distances must control with the highest precision via distance meter [11].

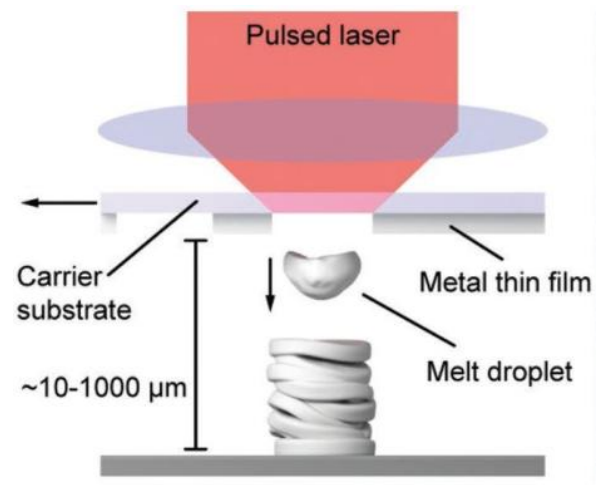

Figure. 5 LITF process schematic [10].

LIFT process has uncountable applications in MEMS and other related industries. One of the examples of utilization of LIFT is in manufacturing of thermal managers in electronic devices. Figure 6 shows a micro heat exchanger that could being manufactured by means of LIFT process [17]. Its innovative structure provides channels with 3 fold surface which gives better and more efficient heat transfer.
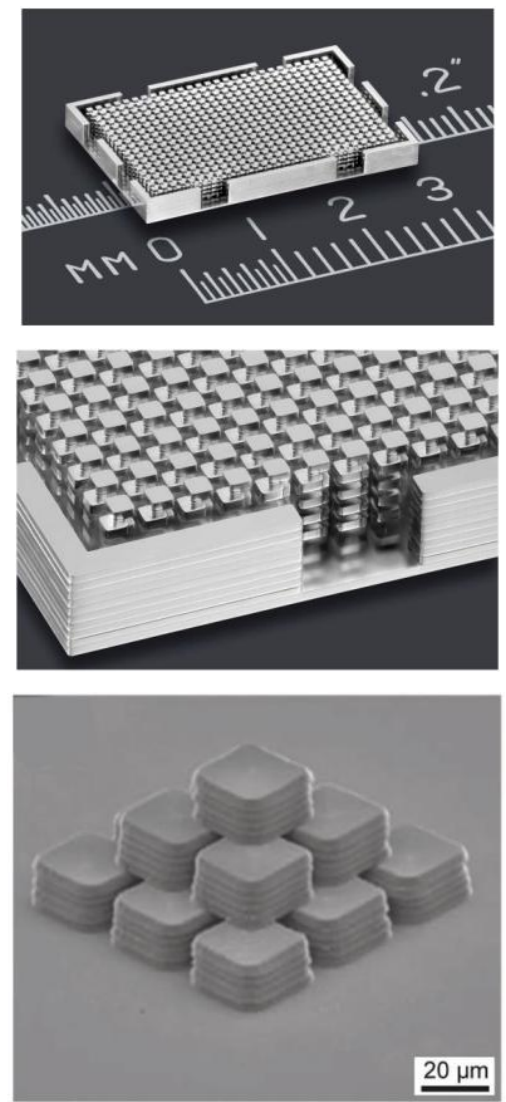

Figure. 6 (Top) thermal heat management system (dimensions: $2 \times 3 \times 0.25 \mathrm{~mm}$, with total surface of $\mathbf{4 3 . 6}$ mm2), (bottom) closer look at pillars $[17,11]$.

There are wide variety of fabricating processes by means of AM and these are only a small fraction of existing methods. Some other methods are laser-assisted electrophoretic 
deposition, electrohydrodynamic printing, laser-induced photoreduction and so on.

\section{BENCHMARK (TEST PIECE ARTIFACT)}

One of the most important prerequisites of utilization of AM as precision manufacturing method is to evaluate process and equipment integrity. This could be achieved via two major methodologies: (1) through a series of direct measurements of machine and process characteristics and (2) through measurements of manufactured test artifacts [18] by methods like X-ray computed tomography (XCT) [19] or CMM [20]. Prior methodology is associated with condition monitoring (CM) methods such as acoustic emission (AE), closed loop control systems, high speed cameras, etc. Later methodology is related to build a part with various features such as holes, notches, bosses, tubes, angles, etc., in different shapes to evaluate capabilities and limitations of an AM system as well as to apply system improvements by linking specific errors measured in the test artifact to specific sources in the AM system [18]. An example of such test pieces is shown in figure 7. Such benchmark includes several features like flat base, tubes, cones, cylindrical holes, angled surfaces, spheres, hollow cube and cylinders. Several researchers work on optimum structure of benchmarks [21, 22]. However, there is no standardized artifact to detect all the faults that could occur during the AM processes. According to [18] the smallest features could be achieved by selective laser melting (SLM) process is $0.2 \mathrm{~mm}$ thin wall and $0.25 \mathrm{~mm}$ cylindrical section diameter. As shown in figure 8, SLM machine fails to build top side of the lateral cylindrical hole and hollow cube.

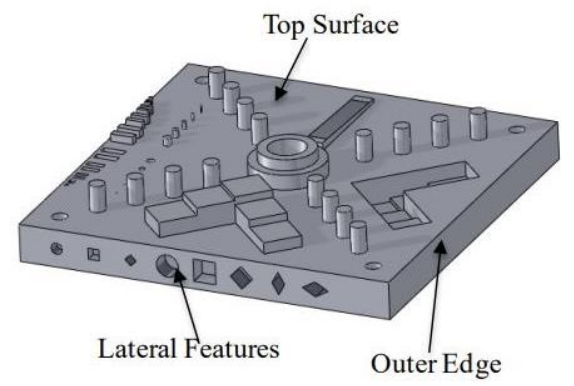

Figure. 7 An example of test artifact with different features [18].

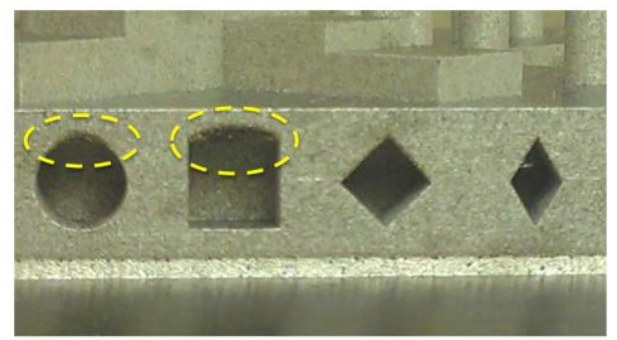

Figure. 8 SLM deficiencies in manufacturing highlighted features [18].

\section{OPTIMIZATION FOR PRESICION ADDITIVE MANUFACTURING}

To utilize AM as a precision manufacturing process, effective parameters should be identified and optimized for lower porosity and surface roughness, better dimensional accuracy, lower thermal gradient, cooling rate, etc. These parameters include: process type, laser diameter, scan strategy, type and size of powder particles, size of melting pool, etc. Attempts are made to achieve optimum process and parameters setup $[23,24]$, however, there is no reliable standard yet. For example, in [23], authors develop a neural network based geometry compensation to predict part deformation after solidification which is lowering the part geometrical accuracy and limit that by the use of optimum build orientation. Another solution is to shorten laser beam to avoid large HAZ and melting pool. Authors in [24] used ultra-short laser pulses to fabricate micro-scale parts by means of stereolitography. This requires very close control on process parameters. Another solution for more precision and integrity could be achieved by hot isostatic pressing (HIP) process [25]. That leads to higher density and accuracy by lowering the porosity inside the part. To predict process output, more accurate models are needed. In some cases, part boundaries could be fabricated with more conservative parameters. For example, laser power could be decreased to create smaller melting pool or lower layer thickness. This strategy may also lead to better tensile and fatigue properties.

\section{PROCESS MONITORING AND NON- DESTRUCTIVE TESTING}

Monitoring and qualification are two important factors in fabricating precision parts. There are several monitoring and evaluating systems such as high-speed digital camera systems, scanning electron micrographs (SEM), micro-computed tomography $(\mu \mathrm{CT})$, laser $\mathrm{CMM}$, etc. For example, Khademzadeh et al. [26] used both $\mu \mathrm{CT}$ and SEM to evaluate micro porosity and micro-scale dimensional accuracy of parts produced by $\mu \mathrm{DMD}$ process. One of the important factor in the use of CT is the effect of surface roughness on dimensional measurements. This leads to a systematic error, however, CT scan seems to be the only way to evaluate both dimensional accuracy and internal defects level. According to [27], internal diameters are larger than CMM reference while external dimensions are smaller. In case of processes such as inkjet or LIFT, the need of close process monitoring is major concern. In most cases, high-speed cameras take care of process monitoring and parameters control.

\section{DISCUSSION}

Conventional additive manufacturing processes such as SLM have some deficiencies which make them inappropriate in case of micro-scale fabrication. However, new process developments provide micro-scale part fabrication by means of AM and such processes have found their position among conventional processes in high-tech industries. Nowadays, term "micro-AM" become common in micro-scale industry, however, more studies are needed to improve micro-AM systems and their production quality. Processes such as inkjet or LIFT have major potential and capability for even nanoscale fabrication. The rate of production are getting higher and new processes are created for different applications. These methods produce no waste and are more flexible and functional than prior methods. There are some limitations such as disability in fabricating overhang and hollow structures which require additional post-processing.

Neural network and finite element methods provide process optimization means and thanks to them, part distortion and residual stress are now predictable. In-situ monitoring are usually done by means of high-speed camera and provide very useful information about melting pool, geometry of droplets, 
possible defects formation and many other major factors. Moreover, $\mu \mathrm{CT}$ and laser $\mathrm{CMM}$ are powerful tools to evaluate dimensional accuracy and precision of the produced parts.

\section{CONCLUSION}

In this paper, the applicability of $\mathrm{AM}$ as a precision manufacturing process is discussed. Inkjet and LIFT process are described in detail. According to presented evidences, lots of studies are needed to characterize effect of each parameter by itself as well as in combination together to achieve optimum result. Benchmarks are powerful tool to identify process capabilities, but they should be more general. Finer powders should be produced for better dimensional accuracy. Advanced monitoring systems are a major requirement and more investigations should be done on the use of high speed cameras. Combining AM with subtractive processes might be a good idea in order to achieve higher accuracy and surface finish. It is concluded that AM could be an ideal solution in case of manufacturing precision parts. AM has bright future as a micro-fabrication process, however, there are some deficiencies that should be overcome.

\section{REFERENCES}

[1] Standard Terminology for Additive Manufacturing Technologies ASTM Standard: F2792-12a.

[2] Uhlmann, E., Mullany, B., Biermann, D., Rajurkar, K. P., Hausotte, T., and Brinksmeier, E. 2016. Process chains for high-precision components with micro-scale features. CIRP Ann. - Manuf. Tech. 65, 549 - 572.

[3] Newman, S. T., Zhu, Z., Dhokia, V., and Shokrani, A. 2015. Process planning for additive and subtractive manufacturing technologies. CIRP Ann. - Manuf. Tech. $64,467-470$.

[4] Wang, Z., Liu, R., Sparks, T., Liu, H., and Liou, F. 2015. Stereo vision based hybrid manufacturing process for precision metal parts. Precision Eng. 42, 1 - 5.

[5] Spierings, A. B., Starr, T. L., Wegener, K. 2013. Fatigue performance of additive manufactured metallic parts. Rapid Prototyping J., 19, 88 - 94.

[6] Zhu, Z., Dhokia, V., Nassehi, A., and Newman, S. T. 2016. Investigation of part distortions as a result of hybrid manufacturing. Robotics and ComputerIntegrated Manuf. 3723 - 32.

[7] Afazov, S., Okioga, A., Holloway, A., Denmark, W., Triantaphyllou, A., Smith, S. -A., and Bradley-Smith, L., 2017. A methodology for precision additive manufacturing through compensation. Precision Eng. 50, $269-274$.

[8] Schaub, A., Ahuja, B., Butzhammer, L., Osterziel, J., Schmidt, M., and Merklein, M. 2016. Additive manufacturing of functional elements on sheet metal. Physics Procedie 83, 797 - 807.

[9] Vaezi, M., Seitz, H., and Yang, S. 2013. A review on 3D micro-additive manufacturing technologies. The Int. J. of Adv. Manuf. Technol. 67, 1721 - 1754.

[10] Teh, K. S. 2017. Additive direct-write microfabrication for MEMS: A review. Frontiers of Mech. Eng. 12, 490 509 .

[11] Hirt, L., Reiser, A., Spolenak, R., and Zambelli, T. 2017. Additive Manufacturing of Metal Structures at the Micrometer Scale. Adv. Mat. 29.
[12] Bhushan, B., Caspers, M. 2017. An overview of additive manufacturing (3D printing) for microfabrication. Microsystem Technol. 23, 1117 - 1124.

[13] Zenou, M., Kotler, Z. 2016. Printing of metallic 3D micro-objects by laser induced forward transfer. Optics Express 24, $1431-1446$.

[14] Lau, G. -K., Shrestha, M. 2017. Ink-Jet Printing of Micro-Electro-Mechanical Systems (MEMS) Micromachines 8.

[15] Martin, G. D., Hoath, S. D., and Hutchings, I. M. 2008 Inkjet printing - the physics of manipulating liquid jets and drops. J. of Physics: Conf. Series 105, 012001.

[16] Murr, L. E., Johnson, W. L. 2017. 3D metal droplet printing development and advanced materials additive manufacturing. J. of Mat. Research and Tech. 6, $77-89$.

[17] Jain, A., Cohen, A. 2015. Ultra-Precision Metal Additive Manufacturing for Thermal Management of Microelectronics.

[18] Moylan, S., Slotwinski, J., Cooke, A., Jurrens, K., and Donmez, M. A. 2014. An Additive Manufacturing Test Artifact. J. of Research of the National Institute of Standards and Tech. 119. $429-459$.

[19] Carmignato, S., Aloisi, V., Medeossi, F., Zanini, F., and Savio, E. 2017. Influence of surface roughness on computed tomography dimensional measurements. CIRP Ann. 66, 499-502.

[20] Satyanarayana, A., Chauhan, A. S., Pradyumna, R., and Baig, M. A. H. 2017. Applications of LASER Inspection for Precision Components. Materials Today: Proc 4, $1230-1235$.

[21] Rebaioli, L., Fassi, I. 2017. A review on benchmark artifacts for evaluating the geometrical performance of additive manufacturing processes. Int. J. of Adv. Manuf. Tech. 93, $2571-2598$.

[22] Thompson, M. K., Mischkot, M. 2015. Design of test parts to characterize micro additive manufacturing processes. Proc. CIRP 34, 223 - 228.

[23] Chowdhury, S., Mhapsekar, K., Anand, S. 2017. Part Build Orientation Optimization and Neural Network Based Geometry Compensation for Additive Manufacturing Process. J. of Manuf. Sci. and Eng. 140, 1 -15 .

[24] in 't Veld, B. H., Overmeyer, L., Schmidt, M., Wegener, K., Malshe, A., and Bartolo, P. 2015. Micro additive manufacturing using ultra short laser pulses. CIRP Ann. $64,701-724$.

[25] Shamsaei, N., Yadollahi, A., Bian, L., and Thompson, S. M. 2015. An Overview of Direct Laser Deposition for Additive Manufacturing; Part II: Mechanical Behavior, Process Parameter Optimization and Control. Additive Manuf. 2, $12-35$.

[26] Khademzadeh, S., Zanini, F., Bariani, P. F., and Carmignato, S. 2018. Precision additive manufacturing of NiTi parts using micro direct metal deposition. Int. J. of Adv. Manuf. Tech. 96, 3729 - 3736.

[27] Aloisi, V., Carmignato, S. 2016. Influence of surface roughness on X-ray computed tomography dimensional measurements of additive manufactured parts. Case Studies in Nondestructive Testing and Evaluation 6, 104 -110 . 